\title{
Erratum to: Introduction to Cancer Stem Cells: Past, Present, and Future
}

\author{
David Bakhshinyan, Ashley A. Adile, Maleeha A. Qazi, Mohini Singh, \\ Michelle M. Kameda-Smith, Nick Yelle, Chirayu Chokshi, \\ Chitra Venugopal, and Sheila K. Singh
}

Erratum to:

Chapter 1 in: Gianpaolo Papaccio and Vincenzo Desiderio (eds.), Cancer Stem Cells: Methods and Protocols, Methods in Molecular Biology, vol. 1692, DOI 10.1007/978-1-4939-7401-6

Sheila K. Singh, one of the authors of Chapter 1 , had the letters of her name transposed in the original published version of the chapter. Her name has been corrected in the current version of the volume. 